\section{Deficiência visual, auditiva e física: prevalência e fatores associados em estudo de base populacional}

\author{
Visual, hearing, and physical disability: \\ prevalence and associated factors in \\ a population-based study
}

Shamyr Sulyvan de Castro ${ }^{1}$ Chester Luiz Galvão César 1 Luana Carandina ${ }^{2}$ Marilisa Berti Azevedo Barros 3 Maria Cecília Goi Porto Alves 4 Moises Goldbaum 5

\title{
Introdução
}

1 Faculdade de Saúde Pública, Universidade de São Paulo, São Paulo, Brasil 2 Faculdade de Medicina de Botucatu, Universidade Estadual Paulista, Botucatu, Brasil.

3 Faculdade de Ciências Médicas, Universidade Estadual de Campinas, Campinas, Brasil. 4 Instituto de Saúde Secretaria de Estado da Saúde de São Paulo, São Paulo, Brasil.

5 Faculdade de Medicina, Universidade de São Paulo, São Paulo, Brasil.

Correspondência S. S. Castro Departamento de Epidemiologia, Faculdade de Saúde Pública, Universidade de São Paulo.

Av. Dr. Arnaldo 715

São Paulo, SP 01246-904, Brasil. shamyr@usp.br

\section{Abstract}

The aim of this study was to describe the prevalence of disabilities according to demographic and socioeconomic characteristics, as well as to determine the main causes. The research used data from two population-based health surveys in São Paulo State, Brazil, in 2002 and 2003, designed with complex sampling techniques. Interviewees that reported disabilities comprised the study sample, according to the database variables. Prevalence of any disability was 110.8\%o; visual disability, 62\%o; hearing disability, 44\%o; and physical disability, $13.3 \%$. Prevalence rates for disabilities varied according to age, gender, and schooling. Prevalence of hearing and physical disability was higher among men. The principal cause of disabilities was the disease itself. External causes were also one of the causative factors in disabilities. Disabilities increased with age and were more prevalent among women and persons with less schooling, and the principal causes were diseases and injuries.

Disabled Persons; Health Profile; Prevalence; Risk Factors
É possível identificar, na história de diversas sociedades, a descrição de pessoas com deficiências. Silva 1 relata exemplos de pessoas deficientes desde os mais remotos tempos, inclusive nas mitologias grega e romana. No entanto, os deficientes passaram a receber maior atenção somente a partir a II Guerra Mundial, com a volta dos veteranos, muitos deles com deficiências diversas causadas por ferimentos 2 .

A Organização Mundial da Saúde (OMS), por meio da Classificação Internacional de Funcionalidade, Incapacidade e Saúde (CIF), define deficiência como "problemas nas funções ou nas estruturas do corpo, como um desvio significativo ou uma perda" 3 (p. 21). O processo incapacitante pode conduzir à invalidez temporária ou permanente para o trabalho 4 , gerando com isso, prejuízos à população economicamente ativa $\mathrm{e}$ também perdas econômicas diretas. Nos Estados Unidos, em 2004, essas perdas foram estimadas em valores próximos a U\$ 4 bilhões 5 com gastos na área de saúde também próximos a U\$ 3,6 bilhões ${ }^{6}$. No Brasil, o Ministério da Previdência Social (http://creme.dataprev.gov.br/temp/DA TIV01consulta36406113.htm, acessado em 24/ Abr/2007) gastou R \$ 1,14 bilhões no ano de 2005 com aposentadorias por invalidez.

Por fatores diversos, os deficientes apresentam um estado de saúde especial e que requer cuidados médicos com maior freqüência que os 
indivíduos não deficientes. Entretanto, os deficientes não são, salvo algumas raras exceções, alvos de campanhas de melhoria e implementação da saúde.

As pesquisas em saúde têm usado freqüentemente os inquéritos de base populacional, que são um instrumento eficiente no levantamento do estado de saúde de coletividades 7,8,9. Eles diferem dos estudos de demanda, uma vez que levantam informações da população em geral, incluindo os indivíduos que não fazem uso dos serviços de saúde 10,11.

A preocupação com o estado de saúde dos deficientes tem raízes nos movimentos sociais que reivindicam igualdade de direitos às pessoas com deficiência, ganhou maior expressão no recente reconhecimento, por parte da população e das autoridades, dos direitos dos deficientes 12,13. Essa tendência reforçou-se no Brasil com a criação de leis que protegem e garantem os direitos de cidadania dos indivíduos deficientes. Poucas pesquisas no Brasil trabalham com o perfil de saúde segundo as características sócio-econômicas dos deficientes 14 .

A determinação das prevalências das deficiências na população e a definição do perfil dos deficientes podem evidenciar a verdadeira face desse grupo de indivíduos. Esses dados podem orientar a assistência a essas pessoas, fornecendo base sólida para planejamento de políticas públicas de saúde, otimizando gastos e melhorando serviços. A determinação das principais causas das deficiências pode fornecer suporte e informações para que sejam feitos trabalhos preventivos e de controle, visando evitar a incidência das deficiências e também implementar seu tratamento e reabilitação.

O objetivo do presente estudo é descrever as prevalências de deficiências segundo características demográficas e sócio-econômicas, bem como indicar suas principais associações com as possíveis causas, analisando os dados de dois inquéritos de saúde de base populacional realizados no Estado de São Paulo, Brasil.

\section{Metodologia}

Os dados analisados são parte de dois inquéritos realizados no Estado de São Paulo: (1) Inquérito de Saúde no Estado de São Paulo (ISA-SP), realizado em 2002 pela Faculdade de Saúde Pública da Universidade de São Paulo (FSP-USP), Faculdade de Medicina de Botucatu da Universidade Estadual Paulista (UNESP) e Universidade de Campinas (UNICAMP); e (2) Inquérito de Saúde no Município de São Paulo (ISA-Capital), realizado em 2003 pelas mesmas instituições responsá- veis pelo ISA-SP. São estudos transversais de base populacional, realizados por meio de entrevistas domiciliares.

Em ambos foi utilizada amostragem em dois estágios. As unidades primárias de amostragem são setores censitários e as unidades secundárias, os domicílios. Para o sorteio da amostra os setores censitários foram agrupados em três estratos com base nos percentuais de chefes de família com nível universitário: menos de 5\%, 5\% a $24,9 \%$ e com $25 \%$ e mais. Foram definidos oito domínios amostrais por sexo e idade, para os quais se planejaram números iguais de entrevistas: menor de 1 ano, 1 a 11 anos, mulheres de 12 a 19 anos, homens de 12 a 19 anos, homens 20 a 59 , mulheres 20 a 59, homens com 60 anos e mais e mulheres com 60 anos e mais.

Neste estudo foram utilizados os dados referentes aos municípios de São Paulo, Botucatu, Campinas e na região sudoeste da Grande São Paulo, Taboão da Serra, Embu, Itapecerica da Serra.

Foram realizadas 8.316 entrevistas, tendo sido identificadas 1.251 pessoas que referiram deficiências. Foram relatadas deficiências físicas (paralisia ou amputações), visuais (baixa visão, cegueira unilateral e cegueira bilateral) ou auditivas (baixa audição, surdez unilateral e surdez bilateral). Ao entrevistado foram feitas as seguintes perguntas: O senhor(a) tem...: (1) dificuldade de enxergar mesmo com óculos/lente?; (2) cegueira de um olho?; (3) cegueira de dois olhos?; (4) dificuldade de ouvir?; (5) surdez de um ouvido?; (6) surdez de dois ouvidos?; (7) paralisia total ou parcial de membros? e (8) perda total ou parcial de membros?

Além da prevalência de deficiências autoreferidas, foram também obtidas estimativas referentes a variáveis sócio-demográficas (sexo, idade, escolaridade do chefe da família e estado civil, migração, raça e situação de trabalho do chefe da família), às causas auto-referidas das deficiências (doenças, causas congênitas, causas externas, envelhecimento e outras); e às dificuldades no trabalho trazidas pela deficiência.

O protocolo da pesquisa foi aprovado pelo Comitê de Ética da Faculdade de Saúde Pública da Universidade de São Paulo. Os entrevistados assinaram um Termo de Consentimento Livre e Esclarecido no qual eram explicados os objetivos da pesquisa e as informações que seriam solicitadas, sendo assegurados o anonimato e a confidencialidade dos dados obtidos.

Para assegurar o controle de qualidade da coleta de dados, as informações de cerca de $10 \%$ dos questionários foram verificadas por nova entrevista. 
Os dados foram analisados através do módulo survey do Stata, versão 9.2 (Stata Corp., College Station, Estados Unidos), tendo sido levados em consideração os distintos aspectos do plano complexo de amostragem: estratificação, sorteio de conglomerados e ponderação. Os pesos foram introduzidos para compensar as diferentes probabilidades de seleção aplicadas à população de estudo e ajustar a amostra à distribuição da população por idade e sexo, segundo dados do Instituto Brasileiro de Geografia e Estatística (IBGE; http://www.ibge.gov.br).

Foi usada a regressão de Poisson 15, adotando-se o nível de significância de 0,20 (teste t) para inclusão da variável no modelo, e de 0,05 (estatística F) para a significância do modelo.

\section{Resultados}

Foram analisados neste estudo os dados de 8.316 pessoas de todas as faixas etárias, incluídas nas amostras dos inquéritos ISA-SP e ISA-Capital. A prevalência de alguma deficiência na população estudada foi de $110,8 \%$, sendo $108,1 \%$ nos homens e 113,2\%o nas mulheres (Tabela 1). A presença de deficiência aumentou com a idade e as prevalências foram mais elevadas no sexo masculino, comparado com o sexo feminino, apenas nas faixas etárias de menores de 12 anos e nas pessoas com 60 anos ou mais ( $p<0,0001$ ). Os menores de 12 anos do sexo feminino referiram a menor prevalência de "alguma deficiência” (21,6\%o) e os homens com 60 anos ou mais o maior valor $(353,9 \%$ o). Do grupo de deficientes estudados, 90\% tinham uma deficiência, 9,5\% apresentaram duas e $0,4 \%$ apresentaram três ou mais deficiências (dados não mostrados em tabela).

Entre os tipos de deficiências referidas, as visuais foram as mais prevalentes $(62 \%$ ) seguidas pelas auditivas (44\%o) e pelas físicas $(13,3 \%$ ) . A dificuldade de enxergar foi a principal deficiência visual referida; a cegueira de um olho e, principalmente, a de dois olhos ocorreram com baixa freqüência. Da mesma forma, na deficiência auditiva, a dificuldade auditiva foi a principal, sendo a surdez unilateral e a bilateral pouco freqüentes. A paralisia de membros foi a principal deficiência física referida, sendo que a perda de membros ocorreu com menor freqüência em todas as idades.

Além de aumentarem com a idade, as prevalências de deficiências revelaram-se associadas ao sexo e à escolaridade do chefe da família, não tendo sido verificadas diferenças quanto à cor/ raça nem quanto ao estado conjugal (Tabela 2). As análises mostraram não haver associação com a naturalidade da pessoa ou com a situação de trabalho do chefe da família (dados não apresentados na tabela).

A razão de prevalências (RP) de deficiência visual é da ordem de 13,4; a de deficiência auditiva 18,8; e a de deficiência física 4,0; tomando como referência o grupo etário com menos de 12 anos comparado ao grupo com 60 anos ou mais.

A deficiência auditiva referida por sexo foi significativamente mais prevalente nos homens $(\mathrm{RP}=1,6)$; assim como a deficiência física $(\mathrm{RP}=$ $2,3)$; enquanto que a visual foi menos prevalente entre os homens $(\mathrm{RP}=0,6)$.

A prevalência de deficiência visual, ajustada por idade e sexo, foi menor entre as pessoas cujo chefe de família apresentava escolaridade de 4 a 7 anos e de 12 anos ou mais, já a deficiência auditiva mostrou-se menos prevalente no grupo cujo chefe de família apresentava escolaridade inferior a 4 anos. Não houve associação da escolaridade do chefe da família com deficiência física.

As causas atribuídas para as deficiências variaram de acordo com a natureza do problema e com o sexo do entrevistado (Tabela 3). A principal causa atribuída de deficiência visual, em ambos os sexos, foi doença: $26,8 \%$ para os homens e $32,1 \%$ para as mulheres. A principal causa da deficiência auditiva foi o grupo das causas externas: $40,2 \%$ entre os homens e, entre as mulheres, $23,9 \%$. A principal causa das deficiências físicas foram as doenças no sexo feminino $(57,2 \%)$; e entre os homens predominaram as causas externas em 46,5\% ( $\mathrm{p}<0,05)$. O envelhecimento, freqüentemente associado a estados incapacitantes 16,17,18, foi citado como causa das deficiências pelos entrevistados, uma vez que não era objeto deste estudo a confirmação clínica e sim a percepção de causa, sempre auto-referida.

Quanto às limitações provocadas pelas deficiências, 33,9\% (intervalo de 95\% de confiança - IC95\%: 27,0-1,5) dos deficientes visuais alegaram que a deficiência prejudicou as suas atividades no trabalho. Essa porcentagem foi de $24,5 \%$ (IC95\%: 17,0-4,0) entre os deficientes auditivos e de 62\% (IC95\%: 41,0-79,3) entre os deficientes físicos (dados não apresentados em tabela).

\section{Discussão}

A real prevalência das deficiências é um tema controverso com estatísticas discrepantes em alguns estudos consultados 19,20,21. Alguns dos fatores que contribuem para essa controvérsia são atribuídos às diferentes definições adotadas e de suas variações: unilateral, bilateral, diminuição da acuidade, paralisia/perda de um membro, 
Tabela 1

Prevalência * de deficiências (\%) segundo sexo e idade, 2002 e 2003.

\begin{tabular}{|c|c|c|c|c|c|c|}
\hline & \multicolumn{4}{|c|}{ Idade (anos) } & \multicolumn{2}{|r|}{ Total [IC95\%] } \\
\hline & Menor 12 & $12-19$ & $20-39$ & $40-59$ & 60 e + & \\
\hline Alguma deficiência (n) ** & $31,7(40)$ & $62,3(127)$ & $87,9(104)$ & $162,2(150)$ & $306,1(830)$ & $110,8(1.251)[95,0-128,0]$ \\
\hline Masculino & 40,8 & 56,4 & 84,1 & 156,0 & 353,9 *** & $108,1[89,0-129,0]$ \\
\hline Feminino & 21,6 & 68,4 & 91,2 & 167,5 & $274,2 * \star \star$ & $113,2[93,0-136,0]$ \\
\hline Deficiência visual $(n)$ ** & $11,7(13)$ & $38,8(77)$ & $45,5(63)$ & $101,4(95)$ & $168,5(471)$ & $62,0(719)[49,0-77,0]$ \\
\hline Dificuldade de enxergar & 11,8 & 36,6 & 43,4 & 97,8 & 151,7 & $58,3[46,0-73,0]$ \\
\hline Cegueira de um olho & 0 & 2,5 & 3,1 & 3,0 & 20,3 & $4,13[2,5-6,7]$ \\
\hline Cegueira de dois olhos & 0 & 0,3 & 0,11 & 2,8 & 1,2 & $0,7[0,1-3,9]$ \\
\hline Deficiência auditiva $(n)$ ** & $9,2(11)$ & $19,9(46)$ & $33,3(33)$ & $54,1(52)$ & $163,3(420)$ & $44,0(562)[36,0-53,0]$ \\
\hline Dificuldade auditiva & 9,2 & 12,69 & 32,3 & 29,6 & 98,3 & $30,7[23,0-39,0]$ \\
\hline Surdez um ouvido & 0,0 & 4,5 & 0,7 & 13,7 & 13,6 & $5,2[3,0-8,0]$ \\
\hline Surdez dois ouvidos & 0,0 & 10,3 & 0,0 & 0,5 & 2,5 & $0,5[0,2-1,2]$ \\
\hline Deficiência física $(n)$ ** & $10,7(16)$ & $5,5(12)$ & $11,0(12)$ & $22,1(18)$ & $39,1(107)$ & 13,3 (165) [10,0-21,0] \\
\hline Paralisia de membros & 7,9 & 5,1 & 9,3 & 11,2 & 15,7 & $9,4[5,0-15,0]$ \\
\hline Perda de membros & 2,8 & 0,3 & 16,3 & 7,1 & 11,2 & $3,8[1,8-7,9]$ \\
\hline
\end{tabular}

* Prevalências na amostra ponderada;

** Número na amostra não ponderada;

*** $p<0,05$ entre os sexos.

Tabela 2

Prevalência (\%) de deficiências segundo variáveis sócio-demográficas, 2002 e 2003 *

\begin{tabular}{|c|c|c|c|c|c|c|}
\hline \multirow[t]{2}{*}{ Variáveis } & \multicolumn{2}{|c|}{ Deficiência visual ( $n=719$ ) } & \multicolumn{2}{|c|}{ Deficiência auditiva $(n=562)$} & \multicolumn{2}{|c|}{ Deficiência física ( $n=165$ ) } \\
\hline & Prevalência ** & RP [IC95\%] & Prevalência ** & RP [IC95\%] & Prevalência ** & RP [IC95\%] \\
\hline \multicolumn{7}{|l|}{ Idade (anos) } \\
\hline Menor 12 & 11,8 & $1,0 \star \star \star$ & 9,2 & $1,0 * \star \star$ & 10,7 & $1,0 * \star \star$ \\
\hline $12-19$ & 38,8 & $3,2 * \star \star[1,4-7,3]$ & 19,9 & $2,1 \star \star \star[0,8-5,8]$ & 5,5 & $0,5 * \star \star[0,1-1,7]$ \\
\hline $20-39$ & 45,3 & 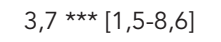 & 33,3 & $3,7 \star \star \star[1,3-10,0]$ & 11,0 & $1,0 * \star \star[0,3-3,4]$ \\
\hline $40-59$ & 101,4 & 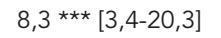 & 54,1 & $6,0 \star \star \star[2,3-15,2]$ & 22,1 & $2,1 * \star \star[0,7-6,0]$ \\
\hline $60 \mathrm{e}+$ & 168,5 & 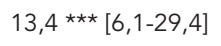 & 163,3 & 18,8 *** $[7,8-45,1]$ & 39,1 & $4,0 * \star \star[1,8-8,0]$ \\
\hline \multicolumn{7}{|l|}{ Sexo } \\
\hline Masculino & 43,3 & $0,6 \#[0,4-0,8]$ & 52,4 & $1,6 \#[1,1-2,3]$ & 21,4 & $2,3 \#[1,2-4,5]$ \\
\hline Feminino & 79,1 & 1,0 \# & 36,4 & $1,0 \#$ & 10,0 & $1,0 \#$ \\
\hline \multicolumn{7}{|l|}{ Estado civil } \\
\hline Solteiro & 34,5 & $1,0 \# \#$ & 20,8 & $1,0 \# \#$ & 15,2 & 1,0 \#\# \\
\hline Casado/Em união & 66,2 & $1,2 \#[0,7-1,9]$ & 51,1 & $1,3 \# \#[0,7-2,3]$ & 14,0 & $0,5 \# \#[0,1-1,4]$ \\
\hline Viúvo/Separado & 119,3 & $1,2 \#[0,7-1,9]$ & 76,4 & 1,4 \#\# $[0,7-2,7]$ & 22,0 & 0,7 \#\# $[0,2-2,2]$ \\
\hline \multicolumn{7}{|l|}{ Escolaridade do chefe } \\
\hline \multicolumn{7}{|l|}{ da família (anos) } \\
\hline 3 ou menos & 103,9 & $1,0 \# \#$ & 34,1 & $1,0 \# \#$ & 13,4 & 1,0 \#\# \\
\hline 4-7 anos & 54,6 & 0,5 \#\#[0,3-0,9] & 44,6 & 1,5 \#\# [0,9-2,3] & 17,5 & 1,4 \#\# $[0,6-3,0]$ \\
\hline 8-11 anos & 65,5 & $0,7 \# \#[0,4-1,2]$ & 45,9 & $1,8 \# \#[1,1-3,0]$ & 15,8 & $1,4 \# \#[0,7-2,7]$ \\
\hline 12 e + & 36,8 & $0,3 \# \#[0,1-0,8]$ & 50,9 & $1,6 \# \#[0,8-2,9]$ & 16,0 & $1,2 \# \#[0,3-3,8]$ \\
\hline
\end{tabular}

* Maior número de não respostas: escolaridade do chefe da família, oito não respostas;

** Prevalências na amostra ponderada;

*** Razão de prevalência (RP) ajustada por sexo;

\# RP ajustada por idade;

\#\# RP ajustada por idade e sexo. 
Distribuição das porcentagens das causas de deficiência segundo tipo de deficiência e sexo, 2002 e 2003.

\begin{tabular}{|c|c|c|c|c|c|c|}
\hline \multirow[t]{2}{*}{ Deficiência } & \multicolumn{6}{|c|}{ Causas das deficiências * } \\
\hline & Doença & Congênita & Externas ** & Envelhecimento & Outros *** & $\begin{array}{c}\text { Não sabe/ } \\
\text { Não respondeu }\end{array}$ \\
\hline Visual & 30,3 & 12,9 & 11,1 & 19,7 & 16,2 & 24,1 \\
\hline Masculino & 26,8 & 16,1 & 15,8 & 17,8 & 17,4 & 22,4 \\
\hline Feminino & 32,1 & 11,4 & 8,7 & 20,6 & 15,6 & 24,9 \\
\hline Auditiva & 17,9 & 9,7 & 33,2 & 15,1 & 16,0 & 25,0 \\
\hline Masculino & 14,4 & 11,7 & 40,2 & 12,1 & 13,8 & 22,0 \\
\hline Feminino & 22,6 & 7,1 & 23,9 & 19,9 & 18,8 & 28,9 \\
\hline Física & 42,9 & 18,5 & 36,9 & 1,6 & 15,1 & 5,4 \\
\hline Masculino & 35,6 & 17,8 & $46,5 \#$ & 1,7 & 14,0 & 8,0 \\
\hline Feminino & 57,2 & 19,8 & $18,3 \#$ & 1,4 & 17,1 & 0,4 \\
\hline
\end{tabular}

* Questão de múltipla resposta, a soma das porcentagens pode ultrapassar 100\%;

** Categoria que agrupa acidentes domésticos, de trabalho, de trânsito e fatores ambientais:

*** Categoria composta por respostas com baixa freqüência, tais como "medicação", "Insolação", "gravidez", entre outras;

$\# \mathrm{p}<0,05$ entre os sexos.

mais de um, impossibilidade de deambulação. Outro fator considerado refere-se à deficiência auto-referida ou aferida, principalmente no caso da deficiência auditiva, onde pode haver subestimação da prevalência quando o dado é coletado de forma auto-referida 22.

A prevalência de pelo menos uma deficiência encontrada na presente pesquisa é maior do que as prevalências de morbidades que são alvo de programas de saúde específicos como artrite/artrose/reumatismo $(78,2 \%$ o); doenças do coração (51,8\%o); e diabetes $(44,4 \%$ o) 11 . Desnecessário dizer que se trata de eventos em saúde diferenciados em relação às deficiências, entretanto, estas últimas podem ter um impacto na saúde e na vida cotidiana das pessoas tão importante quanto as doenças citadas.

A prevalência de algum tipo de deficiência segundo idade e sexo mostrou-se maior para o sexo masculino e faixas etárias extremas, menores de 12 anos e com 60 anos e mais. Três grandes estudos encontraram prevalências de deficiências maiores somente em indivíduos do sexo masculino até por volta dos 14 anos, predominando, depois disso, as freqüências no sexo feminino 23,24,25.

A prevalência geral de deficiência visual aumenta com a idade, conforme registrado em vários estudos e na presente pesquisa 26,27,28,29. Sobre a dificuldade de enxergar, os resultados mostraram que há uma maior prevalência para o sexo feminino a partir dos 12 anos de idade. A respeito da cegueira de um olho e cegueira total (dois olhos), observa-se que houve prevalências variáveis entre os sexos pelas faixas etárias, impossibilitando a identificação de um padrão seguido pelos dados. Merece destaque a maior prevalência de cegueira de um olho no sexo masculino. Uma possível explicação pode ser a relação com os fatores ocupacionais 30 .

As prevalências de deficiências visuais relatadas por diversos estudos com metodologias parecidas variam de $3 \%$ a mais de $300 \%$ om estudos internacionais $31,32,33,34$ e de $7 \%$ o a $382 \%$ o em estudos brasileiros 14,35. Observando-se a RP entre os sexos, expressa-se a maior prevalência entre as mulheres do que nos homens. Picavet \& Hoeymans 31 e Qiu 36 também encontraram maiores prevalências para as mulheres nesse tipo de deficiência. Quando se analisam as prevalências de deficiência visual segundo o nível de escolaridade do chefe da família, percebese maior prevalência no menor nível de escolaridade, o que coincide com dados de outro estudo 31. Esses dados endossam o que alguns estudos apontam: a prevalência de deficiências tem relação com fatores sociais nas coletividades $14,24,25$.

Em relação à prevalência de deficiência auditiva, observa-se aumento com a idade. $\mathrm{Na}$ dificuldade auditiva, surdez unilateral e bilateral percebeu-se que os indivíduos do sexo masculino apresentaram as maiores prevalências quando comparados às mulheres em todas as faixas etárias, resultados semelhantes aos descritos na literatura 31,37 .

Segundo um relatório divulgado pela Coordenadoria Nacional para Integração da Pessoa Portadora de Deficiência (CORDE) \& Associação Fluminense de Reabilitação 14, os valores das 
prevalências de deficiência auditiva de algumas cidades brasileiras variam de $2 \%$ om Taguatinga (Distrito Federal, Brasil) a 30\%o em Porto Velho (Rondônia, Brasil). Em estudos internacionais são relatadas prevalências variáveis de $16 \%$ a $166 \%$ o 25,31,37. A distribuição da prevalência de deficiência auditiva segundo idade mostra que as maiores prevalências acontecem em grupos de idade avançada. Wilson et al. ${ }^{38}$, Picavet \& Hoeymans 31 e Cruickshanks et al. 39 também detectaram esse fato em seus estudos. Ao observar as razões de prevalências, revela-se que os indivíduos entre 20 a 39 anos; os com idade entre 40 e 59 anos e com 60 anos e mais têm mais deficiência auditiva quando comparados aos indivíduos com idade menor que 12 anos.

Conforme já citado, a prevalência de deficiência auditiva é maior no sexo masculino, corroborando Wilson et al. ${ }^{38}$, Picavet \& Hoeymans ${ }^{31} \mathrm{e}$ Cruickshanks et al. ${ }^{39}$. Essa observação pode ser explicada por fatores ligados às categorias profissionais ou maior exposição a fatores de risco.

Tratando-se da deficiência física, observouse que a prevalência também aumenta com a idade, exceção feita para a faixa etária de 12 a 19 anos. Quando a prevalência de deficiência física é analisada segundo o sexo, percebe-se que a prevalência entre os homens é sempre maior do que nas mulheres, o que poderia ser explicado pela maior exposição a fatores de risco, como os ligados a acidentes de trabalho e de trânsito. Os dados observados na Tabela 3 corroboram essa afirmação, apontando as causas externas como a principal causa de deficiência física entre os homens.

A prevalência de deficiências físicas pode sofrer variações de acordo com fatores regionais, Andresen et al. 40 ressaltam o quão diferente as prevalências são dentro dos Estados Unidos, variando de $30 \%$ a $200 \%$. Santos \& Lessa 41 , pesquisando populações faveladas e não faveladas em Salvador (Bahia, Brasil), encontraram prevalências de $62 \%$ o de $18 \%$ o, respectivamente. E, em contraste, com as prevalências apresentadas até agora, no Chile foi verificada uma prevalência de $655 \%$ o de algum tipo de deficiência física ${ }^{25}$. Esses dados mostram a importância dos determinantes sociais, geográficos e de saúde, próprios de cada localidade, no processo incapacitante, uma vez que os estudos citados usaram metodologias parecidas e apresentaram valores divergentes.

Com o aumento da idade, também no caso das deficiências físicas, houve aumento na prevalência. Dados do IBGE em 2000 (Censo demográfico de 2000. http://www.ibge.org.br), Picavet \& Hoeymans 31 e Estudio Nacional de La Discapacidad em Chile 25 também relataram esse comportamento. Depois dos ajustes por sexo verificou-se que na faixa etária 60 anos e mais registrou-se RP de 4,0 quando comparado com a faixa de idade de menores de 12 anos. Essa diferença é decorrente, possivelmente, de maior exposição a fatores etiológicos de deficiência como os acidentes de trabalho, acidentes de trânsito, doenças e outros fatores externos. A análise da distribuição das prevalências de deficiência física conforme o sexo mostrou maior freqüência entre os homens do que entre as mulheres. Essa diferença fica mais clara quando se analisa a RP de 2,3. A maior prevalência de deficiência física entre os homens pode ser justificada pelos fatores relatados acima, sendo que, na presente pesquisa, as causas externas foram, de maneira geral, a segunda maior causa identificada de deficiência física, entre os homens $46,5 \%$ das deficiências foram por causas externas e entre as mulheres somente $18,3 \%$.

A principal causa definida de deficiência visual no presente estudo foi atribuída à doença e, entre os sexos, as porcentagens foram 26,8 para os homens e 32,1 para as mulheres. Como segunda causa de deficiência visual foi relatado o envelhecimento, seguido pelas causas congênitas, em homens e mulheres. A respeito das causas da deficiência visual, os resultados encontrados são semelhantes aos de outros estudos, indicando as doenças como principal causa 25,33,34,42. Alguns autores ainda ressaltam que quase sempre essas doenças são preveníveis ou tratáveis, o que indica que com um melhor acesso aos serviços de saúde a freqüência dessa deficiência provavelmente diminuiria 43,44 . Temporini \& Kara-Jose 45 relatam que estratégias de prevenção da cegueira ou perda visual poderiam ser adotadas nas esferas de prevenção primária (identificação de grupos de risco e proposta de políticas de prevenção); secundária (diagnóstico precoce e atendimento imediato); e prevenção terciária (tratamento e reabilitação de moléstias como a catarata, tracoma, oncocercose, avitaminose A e erros de refração). A idade parece associada à deficiência visual por causa do caráter degenerativo de algumas moléstias advindas com o tempo. As causas congênitas são citadas em outras pesquisas 25,30,34 como causadoras de deficiência visual, porém não com a importância verificada no presente estudo. Nesse caso, cuidados em saúde deveriam ser direcionados às pessoas de grupos de risco para essa deficiência, ou seja, àquelas de idade avançada, com maior ênfase para as mulheres.

A principal causa da deficiência auditiva foram as causas externas. Os deficientes do sexo masculino registraram maior prevalência de deficiências auditivas por causas externas que as mulheres. As doenças foram a segunda maior causa de acometimentos auditivos na presente 
pesquisa e são apontadas como principais causadoras das deficiências auditivas por alguns estudiosos 37,45,46. Hudspeth 46 ressalta a importância do fator ocupacional na etiologia da deficiência auditiva, assegurando que o processo de industrialização e a maior exposição a fatores de risco são importantes na determinação da deficiência auditiva. O fator idade é outro componente importante porque com o envelhecimento os pequenos vasos sanguíneos do ouvido sofrem a ação das placas de aterosclerose, prejudicando a irrigação sangüínea da área, levando a deterioração da audição 46. A prevenção da surdez ou da perda auditiva pode englobar fatores que vão desde a diminuição de tempo de exposição a ruídos, cuidados com medicações ototóxicas, passando por processos educativos voltados à detecção e tratamento das doenças que podem conduzir à perda auditiva 47 .

No caso dos deficientes físicos, as doenças também foram as principais causas. Entretanto entre os homens, as causas externas foram as principais explicações e nas mulheres as doenças. As causas congênitas foram a segunda principal causa entre as mulheres. A maior porcentagem de deficientes físicos do sexo feminino que tiveram como causa da deficiência a doença pode indicar que elas estão mais expostas a fatores incapacitantes ligados a moléstias quando comparadas com os homens, ou seja, mais expostas a doenças que culminam com a deficiência física. Isso poderia ser explicado pela maior longevidade das mulheres, e por isso há uma maior incidência de doenças como acidente vascular cerebral (AVC), artrites e outras doenças incapacitantes.

Chama atenção a presença de acidentes como causadores de deficiências físicas em homens. Pela ordem de importância, os acidentes de trânsito fazem mais deficientes masculinos, seguidos dos acidentes de trabalho. Com relação aos acidentes de trânsito, a maior porcentagem de homens é esperada, frente à maior freqüência desses eventos quando comparados com as mulheres. Em pesquisa feita em Belo Horizonte (Minas Gerais, Brasil), detectou-se que 76,8\% das vítimas fatais de acidentes de trânsito eram do sexo masculino 48 , o que expõe a população sobrevivente aos efeitos desses eventos, dentre eles a deficiência física. Outro estudo, em São José dos Campos (São Paulo) 29, apontou uma porcentagem de $77 \%$ de vítimas do sexo masculino nos acidentes daquela cidade. Boff et al. 49 relatam que em uma amostra de afastamentos de trabalho estudada, $61 \%$ dos afastamentos eram por causa de doenças, $22 \%$ por causas externas (acidentes incluídos) e 17\% por convalescença de cirurgias. As deficiências físicas podem ser evitadas com medidas que impeçam a ocorrência dos fatores desencadeantes do processo que conduz à perda de membros (diabetes, doenças vasculares, acidentes de trabalho etc.) ou à paralisia (acidentes vasculares cerebrais, lesões nervosas, acidentes automobilísticos etc.).

De maneira geral, sem especificar quais deficiências, estudo semelhante feito no Chile 25 mostrou que a principal responsável pelas deficiências também foi a doença em $62,9 \%$ das vezes. As causas congênitas, que foram responsáveis por 9,8\% das deficiências, no Chile representaram a causa de $6,6 \%$. Os acidentes (domésticos, de trabalho e de trânsito) responsáveis por 8,9\% das deficiências em nosso estudo, no Chile representaram 7,7\% das causas de deficiência 25 . Assim, percebe-se que a maioria das causas das deficiências poderiam ser evitadas e combatidas.

As deficiências podem ser importantes fatores incapacitantes para o trabalho. No Canadá o custo total das deficiências em moeda local foi de $\$ 19,2$ bilhões em 1994, sendo que desse total, $\$ 13,6$ bilhões foram com perdas produtivas indiretas, a outra parcela foi gasta em recursos médicos 50 . Entre os indivíduos estudados nessa pesquisa a maior porcentagem de pessoas que tiveram sua capacidade produtiva prejudicada foi entre os deficientes físicos. Snook \&Webster 51 relatam que os gastos com benefícios de trabalhadores afastados por deficiência chegaram a US\$16,1 bilhões nos Estados Unidos no ano de 1986. No ano de 2000 esse valor alcançou a cifra de US\$ 60 bilhões no mesmo país 52 . No Brasil, segundo dados do Ministério da Previdência Social (http://creme.dataprev.gov.br/temp/ DATIV01consulta36406113.htm, acessado em 24/Abr/2007) gastaram-se verbas da ordem de R\$ 1,14 bilhões com aposentadorias por invalidez e R\$ 38 milhões com benefícios assistenciais para pessoas com deficiência nesse mesmo ano. Por esses dados podemos ter uma noção sobre a importância das deficiências para a economia e a força de trabalho.

Observa-se que a ocorrência de deficiências teve prevalência superior a algumas doenças crônicas, que são alvo freqüente de políticas de prevenção, controle e tratamento por parte das autoridades em saúde. Porém, importância semelhante não é dada à questão das deficiências. Alguns exemplos dessa desigualdade são a artrite/artrose/reumatismo, o diabetes e as doenças do coração. Esses eventos em saúde são acontecimentos diferenciados quando comparados com as incapacidades, entretanto, a magnitude das deficiências neste estudo mostra-se superior à das doenças citadas 11. Apesar dessa magnitude, elas não são devidamente consideradas na formulação de políticas de saúde para o grupo populacional com algum tipo de deficiência. 


\section{Resumo}

O estudo descreve as prevalências de deficiências segundo características demográficas e sócio-econômicas, bem como as suas causas. A pesquisa utilizou dados de inquéritos de base populacional realizados em áreas do Estado de São Paulo, Brasil, em 2002 e 2003, com amostragem estratificada por conglomerados. Os entrevistados que referiram deficiências foram a população estudada segundo as variáveis que compõem o banco de dados. A prevalência de alguma deficiência foi de 110,8\%o; deficiência visual, 62\%o; deficiência auditiva, 44\%o e a deficiência física de 13,3\%o. As prevalências das deficiências variaram com a idade; sexo e escolaridade. A prevalência de deficiências auditiva e física foi maior entre os homens. A principal causa das deficiências foi a doença. As causas externas também foram umas das principais causadoras de incapacidades. As deficiências aumentaram com a idade, foram mais prevalentes em mulheres e em pessoas com menor escolaridade, sendo sua principal causa as doenças e as causas externas.

Pessoas Portadoras de Deficiência; Perfil de Saúde; Prevalência; Fatores de Risco

\section{Colaboradores}

S. S. Castro participou na revisão da literatura, análise dos resultados e redação do artigo final. C. L. G. César colaborou na elaboração do projeto, coordenação da pesquisa de campo, orientação e revisão do artigo final. L. Carandina, M. B. A. Barros e M. Goldbaum contribuíram na elaboração do projeto e revisão do artigo final. M. C. G. P. Alves participou da elaboração do projeto, amostragem populacional e revisão do artigo final.

\section{Agradecimentos}

Ao Conselho Nacional de Desenvolvimento Científico e Tecnológico (CNPq) pela bolsa de mestrado concedida a Shamyr Sulyvan de Castro, processo no. 133707/20040. A Secretaria Estadual de Saúde de São Paulo, Secretaria Municipal de Saúde de São Paulo e Fundação de Amparo à Pesquisa do Estado de São Paulo (FAPESP) pelo financiamento para execução da pesquisa pelo projeto de políticas públicas, processo nº. 98/14099-7.

\section{Bibliografia}

1. Silva OM. Epopéia ignorada: a pessoa deficiente na história do mundo de ontem e de hoje. São Paulo: Centro São Camilo de Desenvolvimento em Administração da Saúde; 1987.

2. Rede SACI: Solidariedade, Apoio, Comunicação e Informação. A pessoa cega no processo histórico: um breve percurso. http://www.saci.org.br/index. php?modulo $=$ akemi\&parametro $=17110$ (acessado em 09/Fev/2004).

3. Organização Mundial da Saúde. CIF: classificação internacional de funcionalidade, incapacidade e saúde. São Paulo: Edusp; 2003.

4. Cieza A, Geyh S, Chatterji S, Kostanjsek N, Ustun BT, Stucki G. Identification of candidate categories of the International Classification of Functioning Disability and Health (ICF) for a Generic ICF Core Set based on regression modelling. BMC Med Res Methodol 2006; 6:36.

5. Centers for Disease Control and Prevention. Economic costs associated with mental retardation, cerebral palsy, hearing loss, and vision impairment: United States, 2003. MMWR Morb Mortal Wkly Rep 2004; 53:57-9.
6. Manton KG, Gu X, Lamb VL. Change in chronic disability from 1982 to 2004/2005 as measured by long-term changes in function and health in the U.S. elderly population. Proc Natl Acad Sci U S A 2006; 103:18374-9

7. White KL. Health surveys: who, why and what? World Health Stat Q 1985; 38:2-14.

8. Alves MCGP. Técnicas de replicação em análise de dados de inquéritos domiciliares [Tese de Doutorado]. São Paulo: Faculdade de Saúde Pública, Universidade de São Paulo; 2002.

9. Viacava F. Informações em saúde: a importância dos inquéritos populacionais. Ciênc Saúde Coletiva $2002 ; 7: 607-21$.

10. Cesar CLG, Laurenti R, Buchala CM, Figueiredo MG, Carvalho WO, Caratin CVS. Uso da Classificação Internacional de Doenças em inquéritos de saúde. Rev Bras Epidemiol 2001; 4:120-30.

11. Cesar CLG, Carandina L, Alves MCGP, Barros MBA, Goldbaum M. Saúde e condição de vida em São Paulo: Inquérito Multicêntrico de Saúde no Estado de São Paulo - ISA-SP. São Paulo: Edusp; 2005. 
12. Smith GD, Bartley M, Blane D. The Black report on socioeconomic inequalities in health 10 years. BMJ 1990; 301:373-7.

13. Lawthers AG, Pransky GS, Peterson LE, Himmelstein JH. Rethinking quality in the context of persons with disability. Int J Qual Health Care 2003; 15:289-99.

14. Coordenadoria Nacional para Integração da Pessoa Portadora de Deficiência; Associação Fluminense de Reabilitação. Relatório sobre prevalência de deficiências, incapacidades e desvantagens. Niterói: Associação Fluminense de Reabilitação; 2004.

15. Barros AJD, Hirakata VN. Alternatives for logistic regression in cross-sectional studies: an empirical comparison of models that directly estimate the prevalence ratio. BMC Med Res Methodol 2003; 3:21-34.

16. Guralnik JM, Fried LP, Salive ME. Disability as a public health outcome in the aging population. Annu Rev Public Health 1996; 17:25-46.

17. Thomason SS. Aging: a common denominator of disability. SCI Nurs 2001; 18:3.

18. Gureje O, Ogunniyi A, Kola L, Afolabi E. Functional disability in elderly Nigerians: results from the Ibadan Study of Aging. J Am Geriatr Soc 2006; 54: 1784-9.

19. Organización Panamericana de la Salud. Programa regional de salud del adulto: rehabilitación. Determinación de prevalecía de discapacidades: manual de encuestas domiciliarias. Washington DC: Organización Panamericana de la Salud; 1990.

20. Department of International Development. Disability, poverty and development. http://www. dfid.gov.uk/pubs/files/disabilitypovertydevelop ment.pdf (acessado em 08/Jan/2006).

21. Dudzik P, Elwan A, Metts R. Disability policies, statistics, and strategies in Latin America and the Caribbean: a review. http://www.iadb.org/sds/doc/ Rev2bEditedDisability-PolicyDudzikElwanMetts. pdf (acessado em 16/Jan/2006).

22. Bagai A, Thavendiranathan P, Detsky AS. Does this patient have hearing impairment? JAMA 2006; 295:416-28.

23. Gobierno de El Salvador. Encuesta para personas con discapacidad, 2000/2001. San Salvador: Gobierno de El Salvador; 2001.

24. Gobierno de Nicaragua. ENDIS: encuesta Nicaragüense para personas con discapacidad, 2003. Managua: Gobierno de Nicaragua; 2003.

25. Estudio Nacional de la Discapacidad en Chile. Primer estudio nacional de la discapacidad en Chile. Santiago: Gobierno de Chile; 2004.

26. Dunzhu S, Wang FS, Courtright P, Liu L, Tenzing C, Noertjojo K, et al. Blindness and eye disease in Tibet: findings from a randomized, population based survey. Br J Ophthalmol 2003; 87:1443-8.

27. Khandekar R, Mohammed AJ, Negrel AD, Riyami AA. The prevalence and causes of blindness in the Sultanate of Oman: the Oman Eye Study (OES). Br J Ophthalmol 2002; 86:957-62.
28. Melese M, Alemayehu W, Bayu S, Girma T, Hailesellasie T, Khandekar R, et al. Low vision and blindness in adults in Gurage Zone, central Ethiopia. Br J Ophthalmol 2003; 87:677-80.

29. Nogueira IC. Relatório sobre acidentes de trânsito, ano 2003. São José dos Campos: Departamento de Serviços de Trânsito, Secretaria de Transportes, Prefeitura Municipal de São José dos Campos; 2003.

30. Tabbara KF, Ross-Regnan D. Blindness in Saudi Arabia. JAMA 1986; 255:3378-84.

31. Picavet HSJ, Hoeymans N. Physical disability in the Netherlands: prevalence risk groups and time trends. Public Health 2002; 116:231-7.

32. Brezin AP, Lafuma A, Fagnani F, Mesbah M, Berdeaux G. Prevalence and burden of self-reported blindness and low vision for subjects living in institutions: a nationwide survey. Health Qual Life Outcomes 2005; 3:1-9.

33. Muñoz B, West SK. Blindness and visual impairment in the Americas and the Caribbean. $\mathrm{Br} \mathrm{J}$ Ophthalmol 2002; 86:498-504.

34. Tabbara KF. Blindness in the eastern Mediterranean countries. Br J Ophthalmol 2001; 85:771-5.

35. Medina NH, Barros OM, Muñoz EH, Magdaleno RL, Barros AJD, Ramos LR. Morbidade ocular em idosos da cidade de São Paulo - SP, Brasil. Arq Bras Oftalmol 1993; 56:276-82.

36. Qiu Z. Disability statistics in the People's Republic of China. http://www.dinf.ne.jp/doc/english/asia/ resource/z00ap/003/z00ap00308.htm (acessado em 12/Jan/2006).

37. Fook L, Morgan R. Hearing impairment in older people: a review. Postgrad Med J 2000; 76:537-41.

38. Wilson DH, Walsh PG, Sanchez L, Davis AC, Taylor AW, Tucker G, et al. The epidemiology of hearing impairment in an Australian adult population. Int J Epidemiol 1999; 28:247-52.

39. Cruickshanks KJ, Tweed TS, Wiley TL, Klein BEK, Klein R, Chappell R, et al. The 5-year incidence and progression of hearing loss: the epidemiology of hearing loss study. Arch Otolaryngol Head Neck Surg 2003; 129:1041-6.

40. Andresen EM, Prince-Caldwell A, Akinci F, Brownson CA, Haggund K, Jackson-Tompson J, et al. The Missouri Disability Epidemiology and Health Project. Am J Prev Med 1999; 16(3 Suppl):63-71.

41. Santos Jr. ACS, Lessa I. Prevalência de incapacidades em dois diferentes grupos sociais em Salvador, Brasil. Bol Oficina Sanit Panam 1989; 106:304-13.

42. Whitcher JP, Srinivasan M, Upadhyay MP. Corneal blindness: a global perspective. Bull World Health Organ 2001; 79:214-21.

43. Esteves JF, Domingues CG, Borges LPK, Skolaude PBV, Bortolomiol L, Muxfeldt RA, et al. Prevalência e causas de cegueira em bairro de Porto Alegre. Arq Bras Oftalmol 1996; 59:244-7.

44. Thylefors B, Negrel AD, Pararajasegaram R, Dadzie KY. Global data on blindness. Bull World Health Organ 1995; 73:115-21.

45. Temporini ER, Kara-Jose N. A perda da visão: estratégias de prevenção. Arq Bras Oftalmol 2004; 67:597-601. 
46. Hudspeth AJ. Hearing and deafness. Neurobiol Dis 2000; 7:511-4.

47. Fausti AS, Wilmington DJ, Helt PV, Helt WJ, Konrad-Martin D. Hearing health and care: the need for improved hearing loss prevention and hearing conservation practices. J Rehabil Res Dev 2005; 42:45-62.

48. Rodrigues Júnior JB, Roque FS, Drumond DAF, Bordoni LS, Oliveira ACMB. Perfil das vítimas fatais de trânsito atendidas no HPS João XXIII. Acta Cir Bras 2007; 17 Suppl 2:22-4.

49. Boff BM, Leite DF, Azambuja MIR. Morbidade subjacente à concessão de benefício por incapacidade temporária para o trabalho. Rev Saúde Pública 2002; 36:337-42.
50. Baldwin ML. Reducing the costs of work-related musculoskeletal disorders: targeting strategies to chronic disability cases. J Electromyogr Kinesiol 2004; 14:33-41.

51. Snook SH, Webster BS. The cost of disability. Clin Orthop Relat Res 1987; (221):77-84.

52. Duddleston DN, Blackston JW, Bouldin MJ, Brown CA. Disability examinations: a look at the Social Security Disability Income System. Am J Med Sci 2002; 324:220-6.

Recebido em 26/Out/2006

Versão final reapresentada em 08/Nov/2007

Aprovado em 13/Dez/2007 